\title{
Place learning in the Morris water task: Making the memory stick
}

\author{
Kevin Bolding and Jerry W. Rudy ${ }^{1}$ \\ Center for Neuroscience, Department of Psychology, University of Colorado, Boulder, Colorado 80309, USA
}

\begin{abstract}
Although the Morris water task has been used in hundreds of studies of place learning, there have been no systematic studies of retention of the place memory. We report that retention, as measured by selective search behavior on a probe trial, is excellent when the retention interval is short (5-10 min). However, performance rapidly deteriorates, so that by $\sim 4 \mathrm{~h}$ the search is no longer selective. Additional experiments revealed that selective search at longer retention intervals was improved by inserting gaps between blocks of training trials, but this effect was a non-monotonic function of the interval separating trial blocks. Our experiments also revealed that the location of the first block of trials (Room A or Room B) was irrelevant to long-term retention. A memory modulation theoretical framework may provide a useful way to understand these findings.
\end{abstract}

Given its complexities, place learning in the Morris water task (Morris 1981) is acquired remarkably fast. Even in the chaos of a student class, laboratory rats learn to navigate directly to the platform in about eight trials, and a few minutes after training, with the platform removed, they will selectively search the training quadrant for the platform. It may not be generally appreciated, however, that although the task is quickly learned, some of the information needed to sustain a selective search for the platform on a probe trial may rapidly degrade or become inaccessible. We have repeatedly observed that when probed $24 \mathrm{~h}$ following a single training session, rats display no preference for the quadrant in which the platform was located during training (J. Rudy, K. Bolding and D. Sprunger, unpubl.).

The experiments we report were motivated by our unpublished repeated failures to obtain good long-term retention following a single training session, and Guzowski's (2002) brief report that retention might be enhanced by separating trial blocks. Considering the enormous popularity of this task for studies of the hippocampus, other brain regions, and memory in general, it is surprising how little is known about the factors that influence its retention. To our knowledge there has been no systematic investigation of this issue.

The goal of this research was to (1) systematically document this forgetting, (2) determine some of the variables that influence long-term retention of place learning, and (3) provide some theoretical insight into the processes that support this memory. In overview, we first established a retention function for rats that received a single, 10-consecutive-trial training session. We confirmed our unpublished observations that there is rapid forgetting of information about the spatial location of the platform. We then conducted a series of analytic experiments designed to shed light on some training variables that increase retention.

\section{Results}

\section{Experiment 1: Retention following consecutive-trial} training

Our unpublished observations indicated that rats trained for 10 consecutive trials in the Morris Water Maze task would exhibit robust selective search when tested a few minutes after training,

\footnotetext{
${ }^{1}$ Corresponding author.

E-mail JRudy@clipr.colorado.edu; fax (303) 492-2967.

Article published online before print. Article and publication date are at http:// www.learnmem.org/cgi/doi/10.1101/lm.146106.
}

but almost no preference for the target quadrant when tested the next day. In Experiment 1, we confirmed these observations and evaluated some retention intervals. The experiment contained five groups distinguished by the retention interval separating training and testing: (1) $10 \mathrm{~min}(n=8)$, (2) $30 \mathrm{~min}(n=7)$, (3) 4 $\mathrm{h}(n=8),(4) 24 \mathrm{~h}(n=7)$, and (5) $48 \mathrm{~h}(n=8)$. All rats were given 10 trials separated by an intertrial interval of $\sim 2$ min.

Figure $1 \mathrm{~A}$ shows that there were no acquisition differences among the groups, $F_{(4,36)}=1.19, P>0.20$. Figure $1 \mathrm{~B}, \mathrm{C}, \mathrm{D}$ provides three representations of selective performance on the retention test. Figure $1 \mathrm{~B}$ is a standard measure and compares percent time spent in the target quadrant against the average time spent in the other three quadrants. Paired $t$-tests on these data revealed a significant preference for the training quadrant at the 10-min, 30$\mathrm{min}$, and 4 -h $(P \mathrm{~s}<0.05)$ retention intervals, but not at the $24-\mathrm{h}$ or 48 -h intervals. However, when we look at the performance of individual rats, we were impressed that this standard measure of selective search underestimates forgetting of place information: In many cases, although the time an individual rat spent in the training quadrant might exceed chance ( $5 \mathrm{sec}$ ), it might also have spent as much or more time in another quadrant, in which case the individual rat did not display selective search of the training quadrant. To capture this outcome, we compared the time each rat spent in the target quadrant with the time it spent in its other most preferred quadrant (Fig. 1C). Paired $t$-tests on these data revealed differences $\left(P_{\mathrm{S}}<0.05\right)$ at the 10 -min and 30-min retention intervals but not at the 4 -h, 24-h, or 48 -h intervals. Figure 1D compares the groups using a difference score derived from the data presented in Figure 1C. An analysis of variance revealed differences among the groups' retention intervals, $F_{(4,33)}=3.83$, $P<0.05$. Fisher's post hoc test indicated that the 48 -h retention condition differed from the 10-min, 30-min, and 4-h conditions, $P<0.05$.

In summary, both the standard comparison of time in the training quadrant against the average time spent in the other quadrants (Fig. 1B) and the novel comparison of time in the training quadrant against time spent in the other most preferred quadrant (Fig. 1C) revealed that selective search diminished as the retention interval increased. The novel comparison proved to be the most sensitive measure because it revealed no selective search even at the 4 -h retention interval. Thus, in the remaining experiments we will base our conclusions on this measure, and the data will be represented using the difference score (time spent in target quadrant-time spent in the other most preferred quadrant). 
A

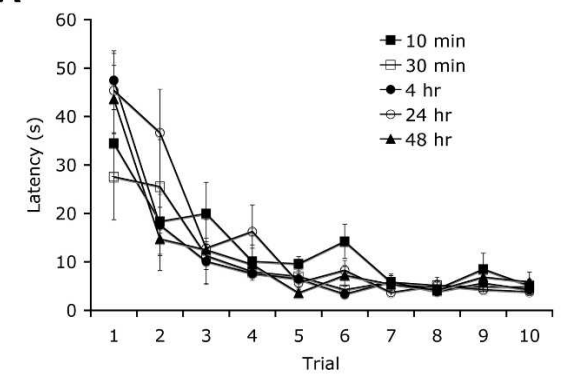

C

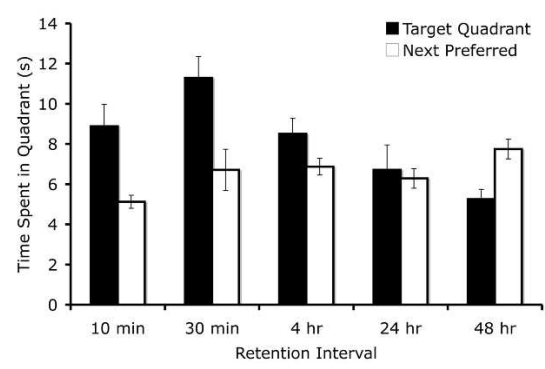

B

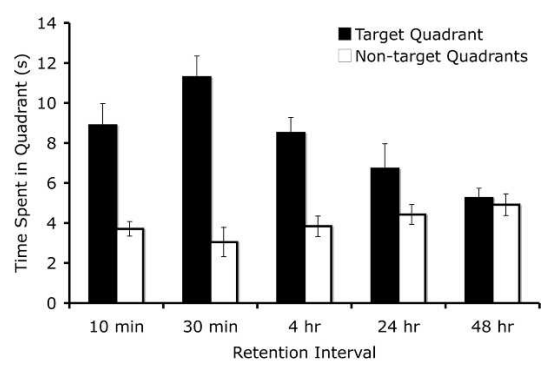

D

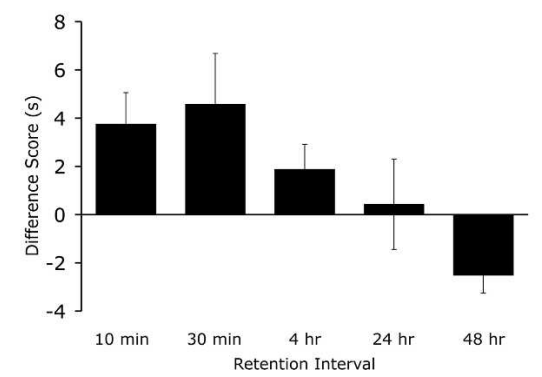

Figure 1. Effect of retention interval on 10-trial water maze performance. $(A)$ Acquisition performance, $(B)$ probe trial performance as measured by comparing time spent in the target quadrant with an average of time spent in all three non-target quadrants, $(C)$ probe trial performance as measured by comparing the time each rat spent in the target quadrant with the time it spent in its next most preferred quadrant. $(D)$ Difference score index of selective search. This measure was obtained by subtracting the time each rat spent in its next most preferred quadrant from the time it spent in the target quadrant. Selective search is indicated by the magnitude of the difference score. A score of 0 indicates no selective search.

\section{Experiment 2: Short-term retention is not disrupted by novel context exposure and depends on the number of trials}

Short-term retention was quite robust. In this experiment we attempted to determine if the memory was held in an active store that would be vulnerable to disruption. In addition, we varied the number of training trials. Rats were given either five or 10 consecutive trials. Immediately following training they remained in the familiar training cage or were placed into a novel environment in a separate room for $5 \min ($ all $n=4)$. The rationale for this manipulation was that the opportunity to explore a novel context between training and retention could potentially displace the active memory of the platform location.

Figure $2 \mathrm{~A}$ shows that rats in all conditions approached asymptote in about five trials and that there were no differences between the two groups that received 10 trials. Figure $2 \mathrm{~B}$ shows that context exploration had no effect on retention, but rats that received 10 training trials spent more time in the training quadrant than rats given only five trials. An ANOVA revealed only a main effect of training trials, $F_{(1,12)}=17.86$. A paired $t$-test indicated rats that received 10 training trials selectively searched the training quadrant.

Allowing the rats to explore a novel context during the 5 min between training and testing had no effect on the rats' retention performance. If the 5-min retention test was supported by an active, working memory trace, one might have expected that exploring a novel environment would have disrupted test performance. Thus, these data provide no evidence for the hypothesis that retention performance was supported by an active memory trace. However, it is possible that our interference manipulation was not sufficient to displace the place memory trace. For example, Ferbinteanu and McDonald (2000) have proposed that representations of places and contexts are somewhat orthogonal, so that it is possible that the subset of active neurons representing the place location is not affected by the activation of the neurons supporting the representation of the recently explored context. These results do, however, provide additional evidence that 10 trials of training were sufficient to support a strong search when the retention interval is short. They also indicate that five trials were not enough training to support a selective search, even with a 5-min retention interval.

We draw two conclusions from the first two experiments. First, with 10 trials (but not five trials) of training, rats acquire the information about the location of the platform needed to support a very selective search when the retention interval is short (5-10 min). We interpret the selective search pattern at the short retention interval at face value-meaning that if the rat has a strong memory of where the platform is located, it will focus its search in that location. Second, if one accepts this interpretation, then it follows that the nonselective search displayed at longer retention intervals means that some of the information contained in the initial memory trace has either degraded or become inaccessible. Nevertheless, it is possible that the nonselective search observed when the retention interval was long occurred because the rat adapted the strategy of abandoning a search of the target quadrant once it discovered the platform was not there. However, then one would have to explain why rats didn't use this strategy when the retention interval was short.

\section{Experiment 3: Temporal gaps between trial blocks influence retention}

The first experiment revealed that rats given 10 trials, with intertrial intervals of $\sim 2 \mathrm{~min}$, display poor long-term retention. Can long-term retention be improved? Guzowski (2002) reported some data suggesting that introducing a temporal gap between trial blocks can enhance performance at long retention intervals. Following Guzowski's lead, in the next two experiments we examined the effect of trial-block spacing. We compared three training conditions. In Experiment 3a, rats in the no-gap (0-h) condition received 10 consecutive training trials $(n=7)$. The training was divided into blocks of five trials for the other two groups. Following the first block of trials, rats in the 2-h condition $(n=8)$ and 24-h condition $(n=8)$ were returned to their home cage for $2 \mathrm{~h}$ and $24 \mathrm{~h}$, respectively, and then brought back to the testing room to complete the second block of five trials. In a second Experiment (3b), we also compared three conditions. The two training blocks were separated by $5 \mathrm{~min}(n=7), 2 \mathrm{~h}$ $(n=9)$, or $6 \mathrm{~h}(n=9)$, and all groups were tested $24 \mathrm{~h}$ after their final training trial. $24 \mathrm{~h}$ after training, rats were given a 20 -sec probe trial with the platform removed.

Figure 3A shows that the groups did not differ during acquisition. Trial-block spacing, however, significantly influenced retention on the probe trial. Figure 3B presents the mean difference score for the three conditions and shows that there were differ- 

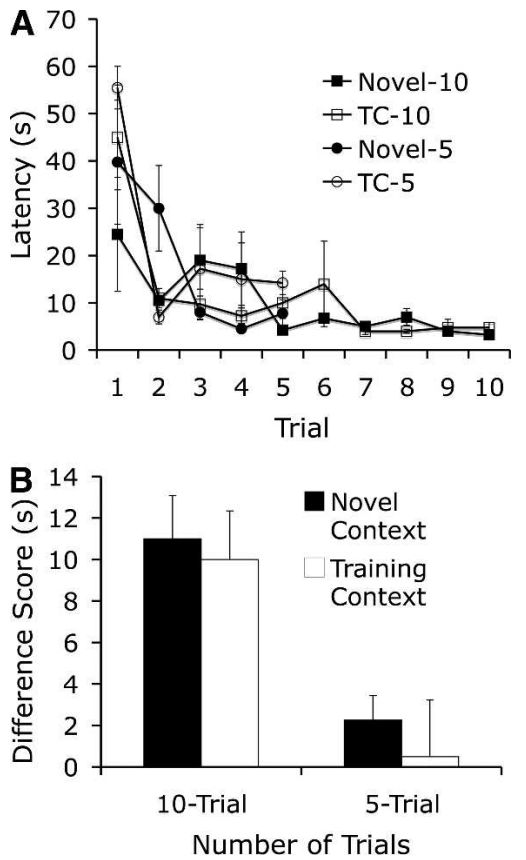

Figure 2. The short-term memory trace is not affected by exposure to a novel environment prior to test. Selective search is observed after 10 training trials, but not after five trials, regardless of post-training condition. $(A)$ Acquisition performance, $(B)$ probe trial performance.

ences among the groups, $F_{(2,19)}=9.66, P<0.01$. Fisher's post hoc analysis indicated that rats in the 2-h spacing condition differed from the other two groups $(P<0.05)$, and that the 0 -h and $24-\mathrm{h}$ group did not differ $(P>0.05)$. Individual paired $t$-tests showed that the 2-h group displayed selective search $(P<0.05)$, but the 24-h and 0-h groups showed no target quadrant preference, with the 0 -h group spending significantly more time in their most preferred non-target quadrant than in the target quadrant ( $P>0.05$ and $P<0.05$, respectively).

Figure $4 \mathrm{~A}$ shows there were no acquisition differences among the conditions of the second experiment, $F_{(2,18)}<1$. However, Figure $4 \mathrm{~B}$ shows there were differences among the conditions on the retention test, $F_{(2,22)}=4.68, P<0.05$. Fisher's post hoc analysis indicated a significant difference between the 2-h and 6-h groups $(P<0.05)$, and a marginally significant difference between the 5 -min and 6 -h groups $(P<0.10)$. The 5 -min and 2 -h group comparison yielded no significant difference $(P>0.05)$. Individual paired $t$-tests indicated that both the 5-min and 2-h groups displayed selective search $(P<0.05)$, but the 6-h group showed no preference for the target quadrant.

Thus, separating the trial blocks by $5 \mathrm{~min}$ or $2 \mathrm{~h}$ yields robust long-term retention, but extending the separation to $6 \mathrm{~h}$ does not support memory enhancement. These results are consistent with Guzowski's (2002) finding that block spacing can benefit long-term retention in the water maze. Our result that a brief 5-min interval facilitates retention is new, as was our finding that the trial-block spacing effect dissipates when extended to $6 \mathrm{~h}$ and $24 \mathrm{~h}$.

\section{Experiment 4: What is the contribution of the initial} trial block?

We knew from unpublished experiments that a session of only five consecutive trials did not produce selective search on a 24-h retention test or 5-min retention test (Experiment 2). The previous experiments demonstrated that, provided trial blocks were separated, the initial five-trial block enhanced 24-h retention performance. So, what is the contribution of the initial trial block to 24 -h retention? Performance certainly improves over the first five trials. So, these trials could contribute to the acquisition of the memory for the specific location of the platform. It is possible, however, that these initial trials contribute to later retention in some nonspecific way, perhaps by altering the rat's state in a way that benefits storage of the information about platform location that was acquired during the last trial blocks (see Guzowski 2002).

The idea that the initial trials initiate some nonspecific processes that can influence later storage makes an interesting prediction. Specifically, it predicts that training in a different room on the first five trials can benefit the storage of the platform location learned in another room, provided the trial blocks are spaced.

To test this hypothesis, two groups of rats were first trained (five trials) in Room A. They received an additional eight trials in Room B, the room used in the previous experiments. We provided three more trials than usual in this experiment to be certain that all rats had fully acquired the platform location in the room they would be tested in. For the 0-h condition, the trials were consecutive with no break between the two blocks of trials. For the 2-h condition, a 2-h gap separated the trial blocks. Both groups were tested $24 \mathrm{~h}$ after their final training trial. Figure 5A shows that there was no difference between the groups during training in Room A or during training in Room B. Increased escape latencies between trials 5 and 6 reflects the change from Room A to Room B. On the probe trial (Fig. 5B), the groups differed $F_{(1,33)}=4.89, P<0.05$, with only the rats given a 2 -h gap between trial blocks displaying selective search.

Even though the initial trial block was administered in a different room, provided there was a gap between trial blocks, it still benefited the storage of the memory for the location of the platform acquired on the second trial block. This means that the initial trial block influences memory storage through some non-
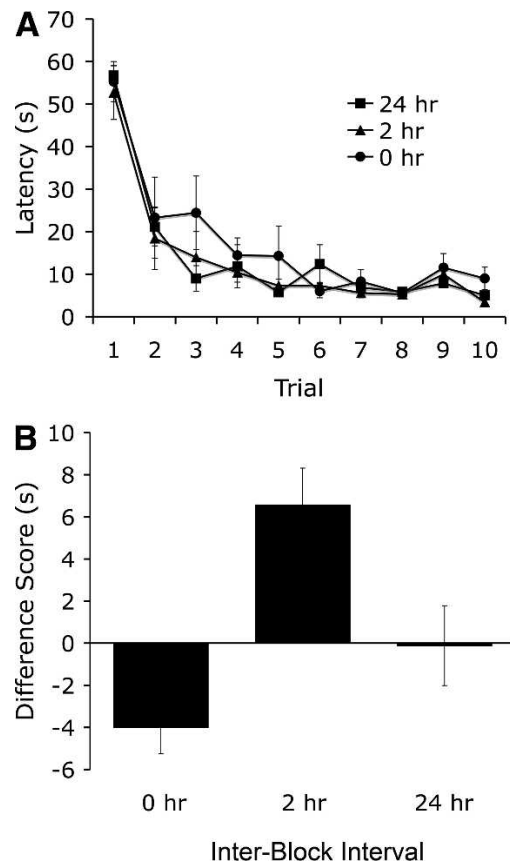

Figure 3. Effect of inter-block interval on long-term retention of platform location. Training blocks separated by $2 \mathrm{~h}$ produce robust long-term retention. $(A)$ Acquisition performance, $(B)$ probe trial performance. 

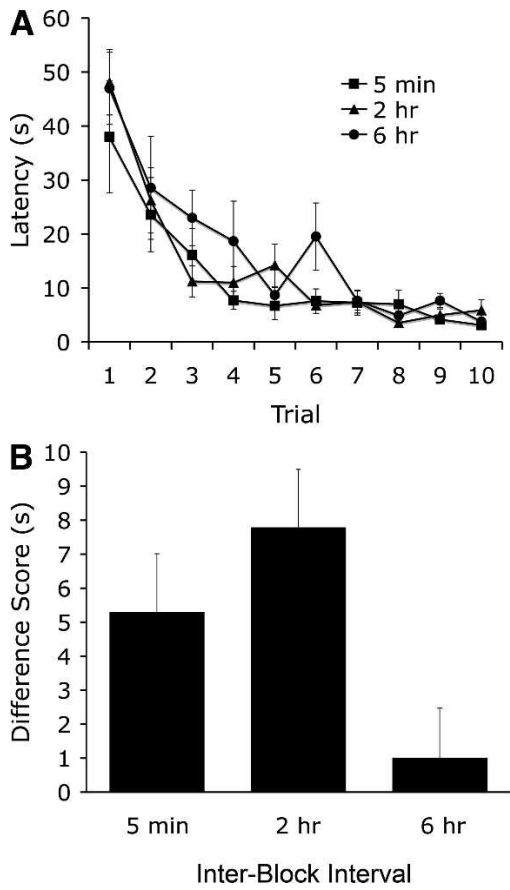

Figure 4. Effect of inter-block interval on long-term retention of platform location. Training blocks separated by 5 min or $2 \mathrm{~h}$, but not $6 \mathrm{~h}$, produce robust long-term retention. (A) Acquisition performance, (B) probe trial performance.

specific processes. We will discuss this issue in detail in the Discussion.

\section{Experiment 5: The role of a context break}

Experiment $3 \mathrm{~b}$ revealed that a 5-min gap between trials 5 and 6 enhanced 24-h retention. This was surprising because this gap is not much different than the interval separating trials 5 and 6 when trials were consecutive in the no-gap condition. There was another difference, however, that may have been more important: Rats in the 5-min group were returned to their home cage in the colony room, whereas rats in the no-gap condition remained in the training cage next to the pool. It might be that just returning the rats to the home cage and not the temporal gap is critical for long-term retention.

In Experiment $5 \mathrm{a}$, we removed this confounding variable to determine whether it was the time between trial blocks or the return to the home cage that enhanced retention. There were three conditions: (1) rats in the consecutive-trial no-gap condition received no break between trial blocks $(n=6),(2)$ rats in the 5 -min HC condition $(n=5)$ were returned to their home cage for the 5 min between trial blocks, and (3) rats in the 5-min TC condition $(n=4)$ remained in the training cage (TC) next to the pool during the $5 \mathrm{~min}$.

As in the previous experiments, there were no differences among the groups during acquisition (Fig. 6A). Figure 6B presents the difference scores for each condition on the probe trial. The between-subjects ANOVA was not significant $(F<1)$. However, the more sensitive within-subjects paired $t$-tests revealed that the 5-min HC condition preferentially searched the target quadrant $(P<0.05)$, but the 5 -min TC and 0 -h groups showed no preference for the target quadrant. These results indicate that it was returning the rats to their home cage between trial blocks, not simply the 5 min interblock interval, that enhanced retention of the platform location.
Given this outcome, in Experiment $5 b$ we determined if the rats had to be returned to their home cage between trial blocks or just removed from the training situation to facilitate retention of the platform location. To do this, one set of rats remained in the training cage during the 5-min break between trials, but the other set was placed into a novel context between trial blocks. Figure 7A shows that there were no differences during acquisition. Figure $7 \mathrm{~B}$ shows that placing the rats into a novel context between trial blocks dramatically enhanced retention of the platform location, $F_{(1,14)}=11.54, P<0.05$. These results indicate that simply removing the rats to a different environment can facilitate retention.

\section{Experiment 6: No effect of alternating training environments}

It is possible that once the rats have reached asymptotic performance, which takes $\sim 5-6$ trials, that the information required to swim directly to the platform is maintained in an active state during the intertrial interval and does not have to be retrieved on each trial. Perhaps having to retrieve the information on each trial facilitates creating a long-term memory that can support selective search. To address this possibility, we created a training protocol in which once the animals were about at asymptote in training room $\mathrm{A}$, we alternated training between Room $\mathrm{A}$ and a new training room, Room $B$. This was done on the assumption that the memory associated with the training trials in Room $\mathrm{B}$ would displace the memory supporting navigation in Room A.

In the first six trials the rats were trained in Room A. Thereafter, trials were given in both Room A and Room B. The exact sequence was AAAAAABBAABBBABABA. Thus, the rats had 11 trials in Room A, approximating the amount of training in other experiments. The acquisition data are displayed in Figure 8. One might have expected that the training in the new context on trials 7 and 8 would have disrupted performance on the subse-
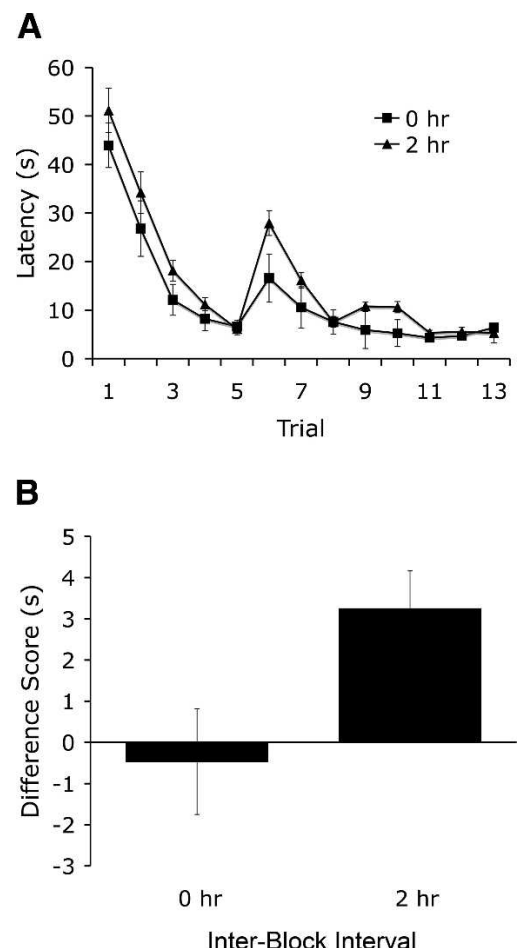

Figure 5. The inter-block interval effect is observed even if the first and second blocks are performed in different rooms. (A) Acquisition performance, $(B)$ probe trial performance. 
A

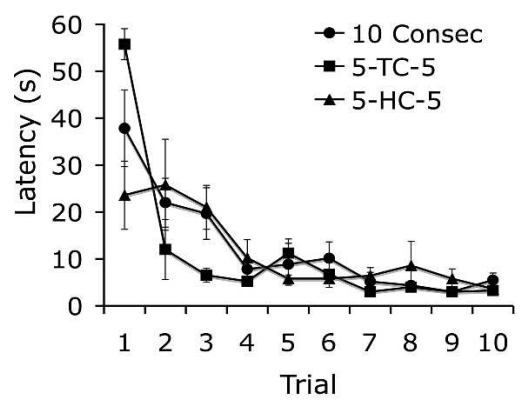

B

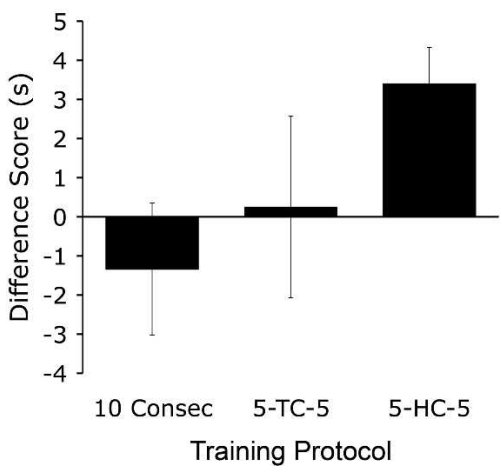

Figure 6. The inter-block interval effect is not observed if animals remain in the training context during the interval. $(A)$ Acquisition performance, $(B)$ probe trial performance.

quent trials in A, but it did not. Moreover, when subsequently probed $24 \mathrm{~h}$ later in Room A, these rats did not display selective search. The average search score was -2.3 . Thus, alternating training rooms did not improve retention. This experiment was predicated on the idea that by alternating training room, the memory for platform location in Room A would be displaced by the memory for the experience in Room B, and that navigation in Room A would depend upon the cues retrieving the memory as opposed to depending on an active trace. Assuming that this indeed occurred, then having to retrieve the memory did not contribute to its long-term stability.

The most surprising outcome of this experiment, however, was that alternating training rooms did not disrupt performance in Room A. Given that performance in Room A was just approaching asymptote, one might have expected that the two trials in Room B would have disrupted subsequent performance in Room A.

\section{Discussion}

The first two experiments established that shortly after 10 consecutive trials of training, rats display a highly selective search for the missing platform, but that retention performance deteriorates rapidly so that by $\sim 4 \mathrm{~h}$ they no longer search selectively. This suggests that (1) an initial memory trace capable of supporting selective search is established, but (2) rapidly degrades or becomes inaccessible. Subsequent experiments revealed that long-term retention could be improved by introducing a temporal gap of 5 min to $2 \mathrm{~h}$ between trial blocks. This gap was effective even when the first trial block was in a different room. Although a gap of $5 \mathrm{~min}$ to $2 \mathrm{~h}$ was effective in enhancing retention, increasing the gap to 6 or $24 \mathrm{~h}$ was not. The temporal gap in the initial experiments was confounded with returning the rats to their home cage. Subsequent experiments revealed that just re- turning the rats to their home cage or a novel environment between trial blocks enhanced retention performance.

Since there has been no previous systematic investigation of retention of place learning, it is difficult to make direct comparisons with other studies. However, we suspect that others have discovered that it is difficult to establish a long-term place memory. This is because it is often the case that when retention intervals of $24 \mathrm{~h}$ or longer are employed, the animals typically receive multiple training sessions administered over several days (see, for example, Sutherland et al. 1982, 2001; Clark et al. 2005; Roozendaal et al. 2004; Martin et al. 2005). Poor search behavior at long retention intervals also might explain why some researchers have resorted to using the so-called Atlantis platform (Spooner et al. 1994) during training to shape the rat to hover over the platform location with the intent of increasing selective search. We point out that such special training is not necessary to get strong selective search behavior when the retention interval is short. We interpret this to mean that if the memory supporting the behavior is strong and accurate, the rat automatically engages in a strong selective search. Thus, the nonselective search that emerges when the retention interval increases reflects a degraded memory trace.

The acquisition and retention of place learning is known to depend on the hippocampus (Morris et al. 1982; Sutherland et al. 2001; Roozendaal et al. 2004; Clark et al. 2005; Martin et al. 2005). Rapid forgetting of a place memory would appear to contrast with what is observed in another task that depends on the hippocampus, contextual-fear conditioning. A single session of training can support retention well over a month (e.g., Kim and Fanselow 1992; Anagnostaras et al. 1999; Frankland et al. 2004). There is of course an important difference between place learning and contextual-fear conditioning. In place learning the animal must use its memory to guide behavior. In the case of maintaining a selective search, a slight degradation in the memory trace could result in a nonselective broadening of the search pattern. In contrast, in contextual-fear conditioning even if the memory

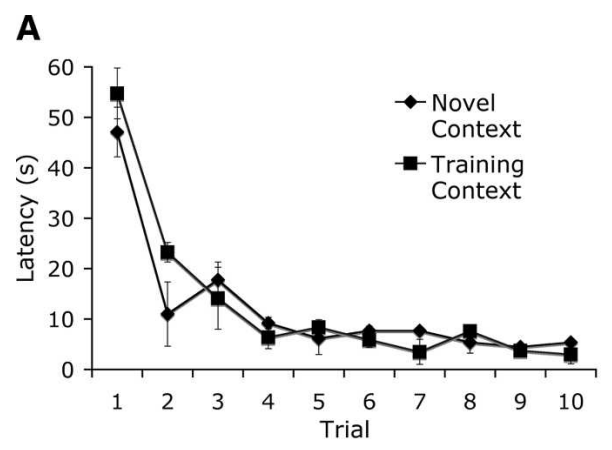

B

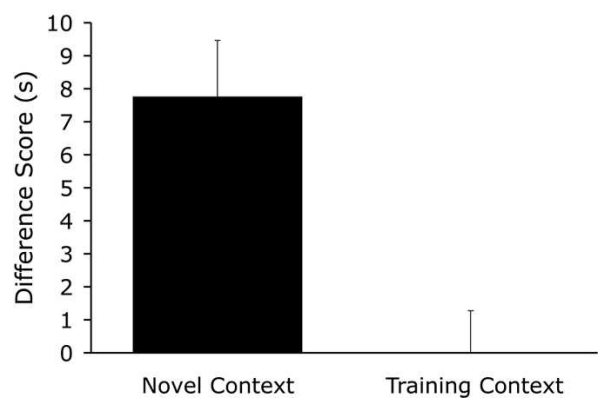

Figure 7. Effect of exposure to a novel environment during a 5-min inter-block interval. $(A)$ Acquisition performance, $(B)$ probe trial performance. 


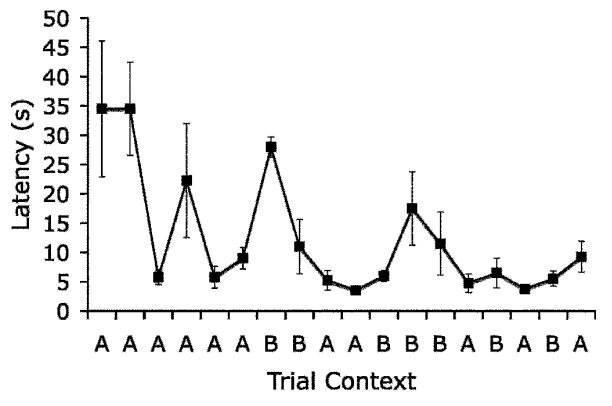

Figure 8. Alternating between two training contexts during acquisition fails to disrupt acquisition performance.

representation is degraded it might still support high levels of fear. In fact, we recently found that forgetting in contextual-fear conditioning is manifested not as a decreased level of freezing, but as a broadening of the generalization gradient (Biedenkapp et al. 2005). Specifically, fear established in one context generalizes more to a similar context as the retention interval increases.

\section{Potential explanations of the gap effect}

Although retention performance rapidly deteriorated following consecutive trials of training, we were able to enhance this memory by inserting a gap between blocks of training. The theoretical challenge is to develop a coherent framework that can explain it. There are several interpretations of the gap effect that can be rejected:

- Massed versus spaced trial effect. The gap effect is just another illustration of the well known principle that retention is better with spaced than massed training.

- Attention/encoding hypothesis. The gap enhances encoding of the information experienced in the second trial block.

- Interference hypothesis. During the initial trials, the rats do not swim directly to the platform and may engage in other behaviors that are incompatible with correct performance. With no gap, these irrelevant and nonspecific activities occurring in the first trial block could produce memory representations that interfered with the consolidation of the relevant information acquired on the second trial block.

- Retrieval failure. This hypothesis assumes that long-term retention following consecutive trials is poor because the cues (internal state cues and external environmental cues) present during training become associated with both relevant and irrelevant information about the location of the platform, and that the subset of cues that is present during testing activates both irrelevant and relevant information. The retrieval of irrelevant information interferes with sustaining a selective search.

In these versions of the interference and retrieval hypotheses, we assume that during the initial trial block (before the rat is swimming directly to the platform) learning occurs that is not necessarily compatible with the learning that mediates performance once the rat is consistently swimming directly to the platform. For example, the rat might learn that the platform is a certain distance from the wall, or that swimming across the pool leads to the platform. We will call this irrelevant learning, and we will call the learning that takes place once the rat is swimming directly to the platform relevant learning.

For over $60 \mathrm{yr}$ it has been recognized that learning and retention are generally better when training trials are spaced than when they are massed (Hilgard and Marquis 1940; Hovland 1951). The gap effect, however, cannot be assumed under this principle for two reasons. First, the function relating retention to gap duration was non-monotonic. When the gap exceeded 6 or $24 \mathrm{~h}$, retention did not improve. Second, in Experiment 5 the gap was held constant at $5 \mathrm{~min}$, but where the rats remained was varied. Briefly returning the rats to their home cages improved retention, whereas leaving them in the training holding cage did not. Thus, there is no obvious way these data can be related directly to the massed versus spaced trial principle.

The attention/encoding hypothesis can be rejected on the grounds that rats trained with no gap searched very selectively when the retention interval was short. This means that the information needed to search selectively was initially encoded. It was with longer retention intervals $(4,24$, and $48 \mathrm{~h}$ ) that the search behavior of rats trained with no gap deteriorated.

The interference hypothesis is consistent with the finding that any short gap ( 5 min to $2 \mathrm{~h}$ ) that involved taking the rats out of the training room enhanced retention. This is because removing them from the room between trial blocks might serve as a distinct marker, keeping the irrelevant memory separated from the relevant memory. This hypothesis, however, ultimately fails because it cannot account for why gaps of 6 and $24 \mathrm{~h}$ did not enhance retention. If the role of the gap was to separate the irrelevant and relevant memories, then these longer intervals should have been the most effective.

The retrieval failure hypothesis is a variation on the interference hypothesis. On this view one would argue that the same cues were present when irrelevant information was acquired during the first trial block and when the relevant information was acquired during the second trial block. Introducing a gap reduced the overlap of these cues so that the cues present when the location of the platform was being learned were distinct from those present at the outset of training and so could retrieve the correct information. This hypothesis assumes that if the cues present during the probe trial are more similar to those that were present on the first trial block than those present during the second trial block, then irrelevant, interfering memories would be retrieved. This hypothesis, however, also has difficulty explaining why gaps of 6 and $24 \mathrm{~h}$ did not enhance retention. If anything, the larger gaps should reduce even further the overlap in cues associated with the location of the platform and cues associated with the irrelevant learning that occurred in the initial trial block.

The elimination of these hypotheses points to the fact that understanding the gap data requires a framework that can deal not only with the importance of the gap, but also the fact that there is a non-monotonicity to the effect. If the gap is too long, it does not benefit retention. This suggests that retention depends on at least two processes. One set of processes is initiated by the initial trials, and another set is generated by the later trials.

\section{Short-term memory and error signals}

We can also image a somewhat more elaborate theory that is based on a combination of two other principles. One principle is that behavior can be mediated by an active, but transient, shortterm memory trace and a retrieved long-term memory (e.g., Wagner 1975). The other principle is that memory storage is regulated, in part, by an error signal that reflects the difference between a predicted outcome and the actual outcome. As this difference decreases, the mechanisms leading to storage become less engaged. This idea is central to standard learning theory (Kamin 1969; Rescorla and Wagner 1972) and recently has been mapped onto brain mechanisms by Schultz and his colleagues (Schultz and Dickinson 2000; Waelti et al. 2001). For example, Waelti et al. (2001) have argued that dopamine, released on occasions when the outcome is not predicted, is an error signal that might contribute to memory consolidation. Moreover, Lisman 
and Grace (2005) have proposed a more general idea that dopamine is released in response to novelty. This is interesting because there is a substantial literature indicating that hippocampus-dependent L-LTP requires not only the activation of NMDA receptors, but also a modulation contribution of dopamine. We reported two experiments that are relevant to this account.

In Experiment 2, we placed the rats in a novel context for 5 min before testing their short-term retention. This manipulation was intended to disrupt the active short-term representation of the platform location, but it had no effect on the short-term retention. Similarly, in Experiment 6 rats were initially trained to near asymptote in Room A and then received alternating trials in a novel room, B. This was done in an attempt to displace the information about the location of the platform in Room A with information about the platform location in Room B. This manipulation did not disrupt acquisition performance in Room A, nor did it enhance long-term retention. Together, these two experiments provide no support for the idea that information in an active short-term memory system was preventing the occurrence of an error signal needed to facilitate storage in long-term memory. The fundamental problem with these experiments, however, was that neither manipulation caused the rat to make errors. Evidently, the memory processes that sustain short-term retention of the platform location are very difficult to disrupt, even though some of the information needed to support search behavior naturally decays within a short period of time.

\section{A modulation framework for establishing a long-term place memory}

One achievement of contemporary neurobiological approaches to memory is the recognition that a behavioral experience can have at least two effects. It can activate (1) synapses that can store the representation of the experience, and (2) more general processes that regulate the storage of properties of those synapses. These ideas first emerged from the work of McGaugh and his colleagues as the concept of memory modulation (for review, see McGaugh 2004). In overview, experience is thought to activate adrenal hormones that then exert an influence on the amygdala, which in turn influences processing in the brain regions that store the memory.

More recently, related ideas can be found in the synaptictagging hypothesis (Frey and Morris 1998) and in speculation about the role of immediate early genes (IEGs) (Clayton 2000; Guzowski 2002). The synaptic-tagging hypothesis argues that the electrical stimulation that can generate long-lasting long-term potentiation (L-LTP) sets in motion two independent processes, (1) the tagging of synapses so that they can capture the plasticity products needed for L-LTP, and (2) a genomic cascade that can produce new plasticity products. Both Guzowski (2002) and Clayton (2000) have proposed that a behavioral experience can induce the transcription of IEGs that can increase a neuron's potential for storing information. The IEGs themselves do not code for experience, but they can facilitate the storage of another experience. These views share the common idea that the storage properties of neurons that represent experience are modifiable.

\section{Application}

We take as axiomatic that a long-term place memory requires that experience activate the relevant synapse needed to represent the place location. The general question is: How does this activation pattern, which can support a transient short-term place memory, get converted to a more stable long-term representation? In principle, the modulation framework provides a way to explain how a gap between training blocks promotes long-term storage. Its value can be appreciated by addressing several other questions raised by the gap effect.

- What is the function of the initial block of training?

- Why is the location of the first set of training trials irrelevant?

- What is the function of the gap?

- Why is taking the animal out of the training environment necessary?

- Why is the function relating the length of the gap to long-term retention non-monotonic?

What is the function of the initial block of training, and why is its location irrelevant?

The answer to these questions is that the fundamental role of the initial training trials is to activate the hormonal and perhaps IEG responses that contribute to long-term storage. This may well depend on the amygdala (Huff et al. 2005a,b; McIntyre et al. 2005). The location of the initial training trials is largely irrelevant because their function is to generate the non-specific hormonal response and allow the rats to learn nonspecific aspects of the task, and not the location of the platform.

\section{What is the function of the gap, and why must the animal be removed from the training room?}

We are still not confident about our answer to this question. However, one possibility is that the modulatory response to the initial trials habituates. Perhaps the removal from the training environment allows the modulatory response to recover and further contribute to memory storage.

\section{Why is the function relating the temporal gap to long-term retention non-monotonic?}

This function is non-monotonic because the memory-enhancing products of the hormonal modulators activated by the initial trials have a limited duration. So, if the gap is too long they will not be available to contribute to the consolidation of the relevant synapses that represent the platform location.

\section{Conclusions}

In conclusion, following a single session of consecutive trials, retention performance in the Morris task as measured by selective search of the training quadrant rapidly deteriorates. Selective search at longer retention intervals was improved by inserting gaps between blocks of training trials, but this effect was a nonmonotonic function of the interval separating trial blocks. A memory modulation theoretical framework may provide a useful way to understand these findings.

\section{Materials and Methods}

\section{Animals}

Adult male Long-Evans rats weighing 250-300 g at the start of the experiments were bred at the University of Colorado. Animals were housed two to four per cage, maintained on a 12-h light/12-h dark cycle (lights on at 6:00 a.m.) at $25^{\circ} \mathrm{C}$, and allowed free access to food and water. Experiments were conducted in accordance with protocols approved by the University of Colorado Animal Care and Use Committee.

\section{Water task apparatus}

The water maze consisted of a circular galvanized steel pool $\sim 17$ $\mathrm{cm}$ in diameter and $58 \mathrm{~cm}$ deep. A movable escape platform, constructed of a Plexiglas base column, having a height of $43 \mathrm{~cm}$, and topped by a round platform $15 \mathrm{~cm}$ in diameter, was placed in one quadrant of the pool and was maintained there throughout acquisition of the task. The water was filled to a height of $47 \mathrm{~cm}$, maintained at $26^{\circ} \mathrm{C} \pm 1{ }^{\circ} \mathrm{C}$, and rendered opaque with non-toxic,

\section{Learning \& Memory}


water-soluble white paint. There were many prominent visual cues that remained constantly positioned around the testing room throughout the study, to serve as distal spatial cues to the location of the platform. No local cues, within the pool, were present. A video camera was mounted on the ceiling to tape the rats' swim patterns during the probe trials.

\section{Behavioral procedures}

\section{Training}

Rats were transported to the training room one cage at a time and were held in a separate cage during training. Each rat received a brief "practice" swim to equalize swimming ability during the first trial. Starting position in the water maze was pseudorandom, and if the rat did not find the platform within $60 \mathrm{sec}$ it was gently guided to the platform. Once a rat reached the platform, its latency to the platform was recorded, and it was allowed to remain on the platform for $10 \mathrm{sec}$ before returning to the training cage. The inter-trial interval was $\sim 2 \mathrm{~min}$.

\section{Testing}

Retention was tested on a probe trial with the platform removed. The rat was placed into the tank and allowed to search for the platform for $20 \mathrm{sec}$. We chose a 20 -sec probe trial because even immediately after training, when rats should have a strong memory of platform location, search behavior very rapidly becomes affected by frustration and within-trial extinction. Twenty seconds was near the maximum that any individual rat would sustain goal-directed search behavior in our experiments. Testing was videotaped and scored for time in each of the four quadrants.

\section{Dependent variables}

During training, the dependent variable was the escape latency, the time it took the rat to find the platform when released into the pool. Our retention measure was derived from a probe trial. The platform was removed from the pool, and the rat was placed into the water and allowed to swim for $20 \mathrm{sec}$. The time spent in each of the four quadrants was recorded. From these data a difference score was derived for each rat by subtracting the time spent in the most preferred quadrant other than the target quadrant from time spent in the target quadrant where the platform had been located during training. A positive difference score indicates selective search of the target quadrant, 0 indicates no preference for the target quadrant, and a negative value indicates a selective search in another quadrant. This is a stringent test for selective search because it requires that each rat spend more time in the training quadrant than in any other quadrant.

\section{Experimental designs}

\section{Experiment 1}

All rats were trained for 10 consecutive trials. At the completion of training they were returned to their home cages in the colony room. $10 \mathrm{~min}, 30 \mathrm{~min}, 4 \mathrm{~h}, 24 \mathrm{~h}$, or $48 \mathrm{~h}$ later they were returned to the testing room and given a probe trial.

\section{Experiment 2}

Rats received either five or 10 trials of training. They either remained in the test environment (TC-5 and TC-10) or were transported to a novel context (Novel-5 and Novel-10), the standard conditioning chamber used in our laboratory to condition fear (see Huff et al. 2005b for description).

\section{Experiments $3 a$ and $3 b$}

In both experiments, all rats received 10 training trials. The three conditions of Experiment 3 a differed by there being either (1) no gap, (2) a 2-h gap, or (3) a 24-h gap between the first and second five-trial blocks. The three conditions of Experiment $3 \mathrm{~b}$ differed by there being either (1) a 5-min gap, (2) a 2-h gap, or (3) a 6-h gap between the first and second five-trial blocks.

\section{Experiment 4}

The first five trials of the experiment were administered in Room A. The second block, eight trials, was administered in Room B. The trials were consecutive for rats in the no-gap (0-h) condition. Trial blocks were separated by $2 \mathrm{~h}$ for rats in the other condition.

\section{Experiments $5 a$ and $5 b$}

In Experiment 5a, there were three conditions: (1) rats in the consecutive-trial no-gap condition received no break between trial blocks $(n=6),(2)$ rats in the 5-min HC condition $(n=5)$ were returned to their home cage for the $5 \mathrm{~min}$ between trial blocks, and (3) rats in the 5-min TC condition $(n=4)$ remained in the training cage (TC) next to the pool during the $5 \mathrm{~min}$.

In Experiment $5 b$, trial blocks were separated by $5 \mathrm{~min}$. Rats in one condition remained in the testing room, but rats in the other condition were taken to an adjacent room and placed into our standard fear-conditioning apparatus for $2 \mathrm{~min}$.

\section{Experiment 6}

For the first six trials, the rats were trained in Room A, the room where the other experiments were conducted. Thereafter, trials were given in both Room A and Room B. Room B was much smaller than Room A and was adjacent to it. This meant that the inter-trial interval was not altered when the training room was switched. The exact training sequence was AAAAAABBAABBBA $B A B A$. Retention for the location of the platform in Room A was assessed $24 \mathrm{~h}$ following training.

\section{Acknowledgments}

This research was supported by National Institutes of Health grant NIH RO1 MH61316 to J.W.R.

\section{References}

Anagnostaras, S.G., Maren, S., and Fanselow, M.S. 1999. Temporally graded retrograde amnesia of contextual fear after hippocampal damage in rats: Within-subjects examination. J. Neurosci. 19: 1106-1114.

Biedenkapp, J., Lopez, D., Sprunger, D., and Rudy, J. 2005. Generalization test reveals rapid forgetting of a hippocampus-dependent contextual-fear memory. Abstract Viewer/Itinerary Planner, Program No. 649.7. Society for Neuroscience, Washington, DC.

Clark, R.E., Broadbent, N.J., and Squire, L.R. 2005. Hippocampus and remote spatial memory in rats. Hippocampus 15: 260-272.

Clayton, D.F. 2000. The genomic action potential. Neurobiol. Learn. Mem. 74: 185-216.

Ferbinteanu, J. and McDonald, R.J. 2000. Dorsal and ventral hippocampus: Same or different? Psychobiology 28: 314-324.

Frankland, P.W., Bontempi, B., Talton, L.E., Kaczmarek, L., and Silva, A.J. 2004. The involvement of the anterior cingulate cortex in remote contextual fear memory. Science 304: 881-883.

Frey, U. and Morris, R.G. 1998. Synaptic tagging: Implications for late maintenance of hippocampal long-term potentiation. Trends Neurosci. 21: 181-188.

Guzowski, J.F. 2002. Insights into immediate-early gene function in hippocampal memory consolidation using antisense oligonucleotide and fluorescent imaging approaches. Hippocampus 12: 86-104.

Hilgard, E.R. and Marquis, D.R. 1940. Conditioning and learning. Appleton-Century, New York.

Hovland, C.I. 1951. Human learning and retention. In Handbook of experimental psychology (ed. S.S. Stevens), pp. 613-689. John Wiley \& Sons, New York.

Huff, N.C., Wright-Hardesty, K., Frank, M., Sprunger, D., Matus-Amat, P., Higgins, E., Biedenkapp, J., and Rudy, J.W. 2005a. Basolateral amygdala influences induction of arc and c-fos mRNA in hippocampus that is associated with contextual fear conditioning. Abstract Viewer/Itinerary Planner, Program No. 316.6. Society for Neuroscience, Washington, DC.

Huff, N.C., Wright-Hardesty, K.J., Higgins, E.A., Matus-Amat, P., and Rudy, J.W. 2005b. Context pre-exposure obscures amygdala modulation of contextual-fear conditioning. Learn. Mem. 12: $456-460$.

Kamin, L.J. 1969. Predictability surprise and attention. In Punishment and aversive behavior (eds. B.A. Campbell and R. Church), pp. 279-296. Appleton-Century-Crofts, New York.

Kim, J.J. and Fanselow, M.S. 1992. Modality-specific retrograde amnesia 
of fear. Science 256: 675-677.

Lisman, J.E. and Grace, A.A. 2005. The hippocampal-VTA loop: Controlling the entry of information into long-term memory. Neuron 46: 703-713.

Martin, S.J., de Hoz, L., and Morris, R.G. 2005. Retrograde amnesia: Neither partial nor complete hippocampal lesions in rats result in preferential sparing of remote spatial memory, even after reminding. Neuropsychologia 43: 609-624.

McGaugh, J.L. 2004. The amygdala modulates the consolidation of memories of emotionally arousing experiences. Annu. Rev. Neurosci. 27: 1-28.

McIntyre, C.K., Miyashita, T., Setlow, B., Marjon, K.D., Steward, O., Guzowski, J.F., and McGaugh, J.L. 2005. Memory-influencing intra-basolateral amygdala drug infusions modulate expression of Arc protein in the hippocampus. Proc. Natl. Acad. Sci. 102: 10718-10723.

Morris, R.G.M. 1981. Spatial localisation does not depend on the presence of local cues. Learn. Motiv. 12: 239-260.

Morris, R.G.M., Garrud, P., Rawlins, J.N.P., and O'Keefe, J. 1982. Place-navigation impaired in rats with hippocampal lesions. Nature 297: 681-683.

Rescorla, R.A. and Wagner, A.R. 1972. A theory of Pavlovian conditioninig: Variations in the effectiveness of reinforcement and nonreinforcement. In Classical conditioning II. Current research and theory (eds. A.H. Black and W.F. Prokasy), pp 64-99. Appleton-Century-Crofts, New York.

Roozendaal, B., Hahn, E.L., Nathan, S.V., de Quervain, D.J., and McGaugh, J.L. 2004. Glucocorticoid effects on memory retrieval require concurrent noradrenergic activity in the hippocampus and basolateral amygdala. J. Neurosci. 24: 8161-8169.

Schultz, W. and Dickinson, A. 2000. Neuronal coding of prediction errors. Annu. Rev. Neurosci. 23: 473-500.

Spooner, R.I.W., Thomson, A., Hall, J., Morris, R.G.M., and Salter, S.H. 1994. The Atlantis platform: A new design and further developments of Buresova's on-demand platform for the water maze. Learn. Mem. 3: $203-211$.

Sutherland, R.J., Kolb, B., and Whishaw, I.Q. 1982. Spatial mapping: Definitive disruption by hippocampal or medial frontal cortical damage in the rat. Neurosci. Lett. 31: 271-276.

Sutherland, R.J., Weisend, M.P., Mumby, D., Astur, R.S., Hanlon, F.M., Koerner, A., Thomas, M.J., Wu, Y., Moses, S.N., Cole, C., et al. 2001. Retrograde amnesia after hippocampal damage: Recent vs. remote memories in three tasks. Hippocampus 11: 27-42.

Waelti, P., Dickinson, A., and Schultz, W. 2001. Dopamine responses comply with basic assumptions of formal learning theory. Nature 412: $43-48$.

Wagner, A.R. 1975. Priming in STM: An information processing mechanism for self-generated and retrieval generated depression of performance. In Habituation: Perspectives from child development, animal behavior and neurophysiology (eds. T.J. Tighe and R.N. Leaton), pp. 95-128. Erlbaum, Hillsdale, NJ.

Received November 23, 2005; accepted in revised form February 10, 2006. 


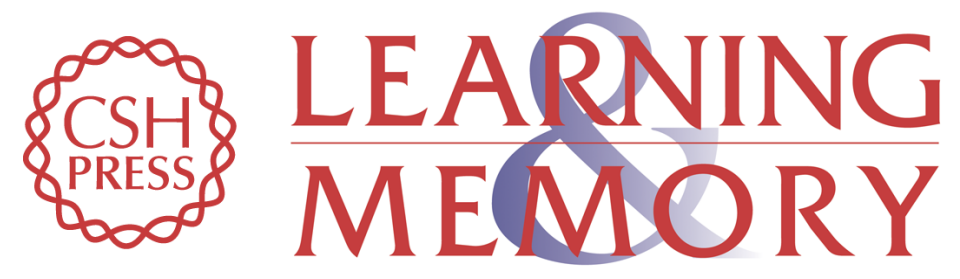

\section{Place learning in the Morris water task: Making the memory stick}

Kevin Bolding and Jerry W. Rudy

Learn. Mem. 2006, 13:

Access the most recent version at doi:10.1101/lm.146106

References This article cites 21 articles, 6 of which can be accessed free at: http://learnmem.cshlp.org/content/13/3/278.full.html\#ref-list-1

License

Email Alerting Receive free email alerts when new articles cite this article - sign up in the box at the Service top right corner of the article or click here. 